\title{
Age and Axillary Lymph Node Ratio in Postmenopausal Women with T1-T2 Node Positive Breast Cancer
}

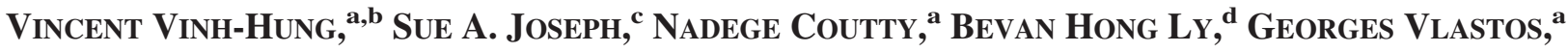 \\ Nam Phong Nguyen ${ }^{\mathrm{e}}$ \\ ${ }^{\mathrm{a}}$ University Hospitals of Geneva, Geneva, Switzerland; ${ }^{\mathrm{b}}$ Vrije Universiteit Brussel, Elsene, Belgium; \\ ${ }^{c}$ University of Northern Iowa, Cedar Falls, Iowa, USA; ${ }^{\mathrm{d} J o h n}$ A Burns School of Medicine, University of \\ Hawaii at Manoa, Honolulu, Hawaii, USA; ${ }^{\mathrm{e} U n i v e r s i t y ~ o f ~ A r i z o n a, ~ T u c s o n, ~ A r i z o n a, ~ U S A ~}$
}

Key Words. Breast neoplasms • Lymph nodes • Prognosis • Survival • Elderly • SEER Program • Competing risks • Cause-specific mortality • Generalized Additive Models for Location Scale and Shape • GAMLSS •

Bayesian Information Criterion • BIC

\section{Disclosures}

Vincent Vinh-Hung: None; Sue A. Joseph: None; Nadege Coutty: None; Bevan Hong Ly: None; Georges Vlastos: None; Nam Phong Nguyen: None.

Section Editor Gabriel N. Hortobagyi discloses that he serves as a consultant for Allergan, Genentech, sanofi-aventis, Novartis, Taivex LLC, and Merck, and received research funding from Novartis.

Section Editor Kathleen Pritchard discloses that she serves as a consultant for and receives honoraria from Novartis, Roche, AstraZeneca, and Pfizer.

Reviewer "A" discloses no financial relationships.

Reviewer "B" discloses no financial relationships.

Reviewer "C" discloses no financial relationships.

The content of this article has been reviewed by independent peer reviewers to ensure that it is balanced, objective, and free from commercial bias. On the basis of disclosed information, all conflicts of interest have been resolved.

\section{LEARNING OBJECTIVES}

After completing this course, the reader will be able to:

1. Identify changes in medical/surgical practice that can affect the prognostic performance of established prognostic factors such as lymph node involvement in breast cancer.

2. Explain how the lymph node ratio can help to adjust against practice-related changes.

3. Use the lymph node ratio as a measure of tumor burden in order to weigh the risk of breast cancer death against other causes of death in elderly patients.

\section{Abstract}

Purpose. The purpose of this article was to examine the relationship between age and lymph node ratio (LNR, number of positive nodes divided by number of exam- ined nodes), and to determine their effects on breast cancer (BC) and overall mortality.

Methods. Women aged $\geq 50$ years, diagnosed in 1988-

Correspondence: Vincent Vinh-Hung, M.D., Ph.D., University Hospitals of Geneva, rue Gabrielle-Perret-Gentil 4, 1211 Geneva 14, Switzerland. Telephone: +41 223827 118; Fax: +41 223827 117; e-mail: anhxang@ gmail.com Received February 17, 2010; accepted for publication August 24, 2010; first published online in The Oncologist Express on October 7, 2010. @AlphaMed Press 10837159/2010/\$30.00/0 doi: 10.1634/theoncologist.2010-0044 
1997 with a unilateral histologically confirmed T1-T2 node positive surgically treated primary nonmetastatic BC, were selected from the National Cancer Institute's Surveillance, Epidemiology, and End Results (SEER). Generalized Additive Models for Location Scale and Shape (GAMLSS) were used to evaluate the age-LNR relationship. Cumulative incidence functions and multivariate competing risks analysis based on model selection by the Bayesian Information Criterion (BIC) were used to examine the effect of age and LNR on mortality. Low LNR was defined as $\leq \mathbf{0 . 2 0}$, mid-LNR $0.21-0.65$, and high LNR $>0.65$.

Results. GAMLSS showed a nonlinear LNR-age relationship, increasing from mean LNR 0.26-0.28 at age $50-70$ years to 0.30 at 80 years and 0.40 at 90 years.
Compared with a 9.8\% [95\% confidence interval (CI) $8.8 \%-10.8 \%$ ] risk of $\mathrm{BC}$ death at 5 years in women aged $50-59$ years with low $L N R$, the risk in women $\geq 80$ years with low LNR was $12.6 \%$ [95\% CI 10.1\%-15.0\%], midLNR 18.1\% [13.9\%-22.1\%], high LNR 29.8\% [22.7\%$36.1 \%$ ]. Five-years overall risk of death increased from $40.8 \%$ [37.5\%-43.9\%] by low LNR to $67.4 \%$ [61.4\%72.4\% ] by high LNR. The overall mortality hazard ratio for age $\geq 80$ years with high LNR was 7.49 [6.54-8.59], as compared with women aged 50-59 years with low LNR.

Conclusion. High LNR combined with older age was associated with a threefold increased risk of $\mathrm{BC}$ death and a sevenfold increased hazard ratio of overall mortality. The Oncologist 2010;15:1050-1062

\section{INTRODUCTION}

Breast cancer is the most common malignancy affecting women, with more than 1 million new cases occurring worldwide annually [1]. The age-specific incidence rate is highest among patients 70 years or older [2,3]. A decrease in breast cancer mortality has been reported in the 20-69 years age groups [4], but has not been observed in the older age groups $[5,6]$. The high breast cancer mortality among elderly patients in these latter reports is troubling, as several studies noted advancing age was associated with more favorable tumor biology or with a more indolent tumor behavior [7-13]. The question of the biological behavior of breast cancer in elderly patients is an important issue that may directly affect treatment decision: if breast cancer is an indolent disease in older women, then less aggressive treatments might be appropriate. If not, refraining or reducing treatments would result in poorer outcome [14]. In either case, treatment decisions must also consider existing comorbidities that are often present in older women [15-18]. Prognostic factors can potentially aid such decisions. Notably, axillary lymph node involvement is considered as one of the most important. Reviews have shown that the ratio of positive to excised nodes (lymph node ratio, LNR) as a measure of nodal involvement consistently improved prognostication over numbers of nodes $[19,20]$. However, the relationship of the LNR with age has scarcely been investigated [21].

We hypothesize that the severity of disease in elderly women has been underestimated, as might result from less performance of axillary staging with age [22-25]. In the present study, we examine how age relates with LNR, and we investigate how these two factors affect survival in women with T1-T2 node positive breast cancer.

\section{MATERIALS AND MethodS}

Women aged $\geq 50$ years diagnosed in 1988-1997 with a unilateral histologically confirmed nonmetastasized T1-T2 (tumor size $\leq 5 \mathrm{~cm}[26,27]$ ) node positive surgically treated first primary breast carcinoma were selected from the National Cancer Institute's Surveillance, Epidemiology, and End Results 9-Registries (SEER release 2009) [28]. Cases with primary tumors extending beyond the breast parenchyma, non-hospital-based records, patients not under active follow-up, undefined tumor size, and undefined numbers of lymph nodes were excluded.

We conducted the analyses in three successive modules. First, we considered how to describe the relationship between age and LNR. Second, we examined their crude impact on breast cancer survival. Third, we considered whether or not the effect of age and LNR on survival was affected by other prognostic factors.

For the description of how age and LNR related, we used the recently developed approach of semiparametric regression based on the Generalized Additive Models for Location Scale and Shape (GAMLSS) [29]. On consideration that the LNR is derived from the respective values of the number of positive nodes (npos) and the number of nodes removed (ntot), that is, LNR = npos/ntot, we also examined how age was related to npos and ntot.

To examine how age and LNR affect breast cancerspecific survival, without covariate adjustment, we used the method of cumulative incidence curves [30]. To examine their effect taking into account other prognostic variables, we used cause-specific survival analysis based on Lunn and McNeil's data augmentation "B" method applied to Cox models [30, 31]. The method gives the same results as conventional analyses in which other causes of death are censored, but with the advantage of using a single survival model. To select which variables would be included in that single model, we scanned candidate variables by stepwise Cox regression using the Bayesian information criterion (BIC) [32]. Variables evaluated were the SEER registry 
area, race, age (categorized as 50-59, 60-69, 70-79, and $\geq 80$ years), marital status (married vs. else), year of diagnosis (1988-1992 vs. 1993-1997), tumor location (medial vs. else), hormone receptor status (both estrogen and progesterone positive, vs. both negative, vs. else), histology (ductal vs. else), histological grade (grade 3-4 vs. else), type of local therapy (mastectomy or breast conserving, with or without radiation therapy), tumor size ( $>2 \mathrm{~cm}$ vs. $\leq 2 \mathrm{~cm}$ ), ntot (10 or more vs. 1-9 lymph nodes examined), npos (1-3 vs. $4-9$ vs. $\geq 10$ positive nodes) [26, 27], and LNR ( $\leq 0.20$ vs. $0.21-0.65$ vs. $>0.65$ ) [33].

All statistical analyses were done using $\mathrm{R}$ version 2.10.1 [34]. GAMLSS used the package "gamlss" [35]. Competing risks analyses by cumulative incidence curves and by Cox models used the package "survival" [30]. Stepwise regression and application of the BIC used the package MASS [36]. Script programming required to implement the analyses was developed in-house; the program is available on request.

\section{RESULTS}

Subjects matching the selection criteria included 17,685 cases from the SEER 9-Registries diagnosed in 1988 1997. At follow-up cutoff date December 31, 2006, the median follow-up for patients still living (censored for death from any cause) was 12.75 years (interquartile range [IQR], $10.6-15.4$ years).

Table 1 summarizes patient characteristics. Larger percentages of married status, black race, negative estrogen receptor, and breast-conserving surgery with or without radiation therapy were noted in the younger women. Decreased percentages of married status, black race, negative estrogen receptor, and radiation therapy were noted in the older women. The relative percentage of more extensive lymph node examination was higher in younger women $(34.2 \% \geq 10$ nodes examined vs. $27.9 \%<10$ nodes examined). This relative percentage was reversed in older women (24.6/27.2 in the 70-79 year old age group, 8.2/ 15.8 in the $\geq 80$ year old age group). The relative percentages of positive lymph nodes were comparable among the different age groups. The relative percentage of high LNR was lower in younger women $(0.86=29.1 \% / 33.9 \%)$. Conversely, it increased in the older women $(1.43=12.6 \%$ l $8.8 \%$ ). The relative percentage of breast cancer deaths vs. deaths from other causes was higher in younger women $(2.77=34.3 \% / 12.4 \%)$, whereas the relative percentage of deaths from other causes was higher in older women $(2.92=22.8 / 7.8)$.

The distributions of the lymph node values npos, ntot, and LNR, without taking into account age, are shown in Figure 1 . The distributions detailed according to age are shown in Figure 2. By all means, npos could be considered constant across all ages-the GAMLSS showed only an imperceptible decrease of the smoothed mean npos from 3.9 at age 50 years to 3.6 at 90 years (Figure 2, left panel, npos). ntot changed more notably with age, declining from a mean of 16.4 at age 50 years, to 16.3 at 60 years, 15.7 at 70 years, 14.3 at 80 years, and 11.6 at 90 years (Figure 2, middle panel, ntot). The LNR changed inversely, increasing at first slowly and then exponentially, from 0.26 at age 50 years, to 0.27 at 60 years, 0.28 at 70 years, 0.30 at 80 years, and 0.40 at 90 years (Figure 2, right panel, LNR).

Results of the competing risks analysis according to age and LNR but without other covariates adjustment are summarized in Figure 3. The specific cumulative incidence curves were coded by the colors red, purple, green, and blue to indicate age group and by solid line, dashed line, and dotted line to indicate LNR category. The combination of the two distinct methods of coding - color and line styletherefore uniquely identified each of the 12 possible age and LNR subgroups. The left panel (A) displays the risk of death from breast cancer. It shows that patients of different age groups but within the same LNR category had comparable probabilities of dying from breast cancer. The right panel (B) displays the risk of death from other causes. It shows that patients in different LNR categories but within the same age groups had comparable probabilities of dying from other causes. There was evidence of nonproportionality shown by the divergence and crossing of curves with the high LNR $\geq 80$ years age group (plain red curve). This could be explained by the small number of patients in that subgroup ( $n=282,12.6 \%$ of 2,238 patients with high LNR; Table 1).

Tables $2 \mathrm{~A}$ and $2 \mathrm{~B}$ focus on the mortality estimates at 5 years of follow-up. Compared with the $9.8 \%$ [95\% confidence interval (CI) $8.8 \%-10.8 \%$ ] risk of dying from breast cancer at 5 years in women aged 50-59 years presenting with low LNR, the risk in women $\geq 80$ years with low LNR was $12.6 \%$ [95\% CI 10.1\%-15.0\%], increasing with midLNR to $18.1 \%$ [13.9\%-22.1\%], and with high LNR to $29.8 \%$ [22.7\%-36.1\%] (section a in Tables 2A and 2B). The risk of dying from other causes increased with age. In women presenting with a high LNR, it was $4 \%[2.0 \%-$ $5.9 \%$ ] at age 50-59 years, increasing to $37.6 \%$ [30.4\%$43.9 \%$ ] at age $\geq 80$ years (section $\mathrm{b}$ in Tables $2 \mathrm{~A}$ and $2 \mathrm{~B}$ ). The overall risk of dying from any cause in women $\geq 80$ years increased from $40.8 \%$ [37.5\%-43.9\%] with low LNR to $67.4 \%$ [61.4\%-72.4\%] with high LNR (section $\mathrm{c}$ in Tables $2 \mathrm{~A}$ and $2 \mathrm{~B}$ ).

We proceeded next to the analyses with covariates adjustment. Considering overall mortality endpoint, age and 


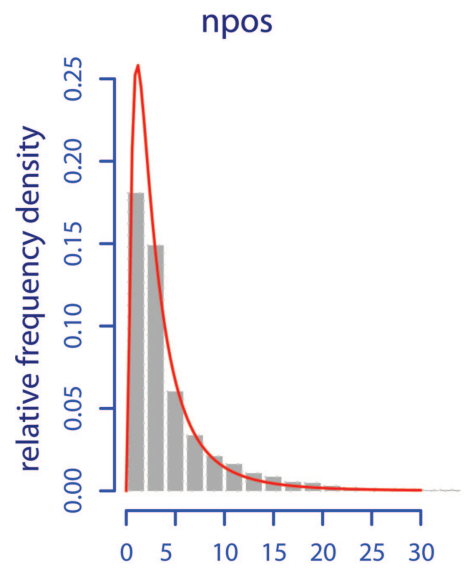

Number of positive nodes

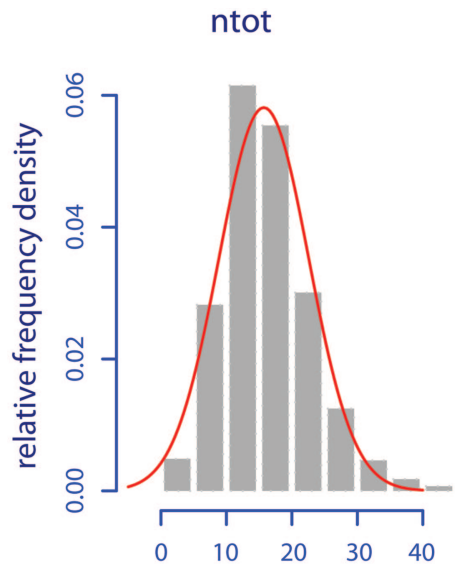

Number of examined nodes

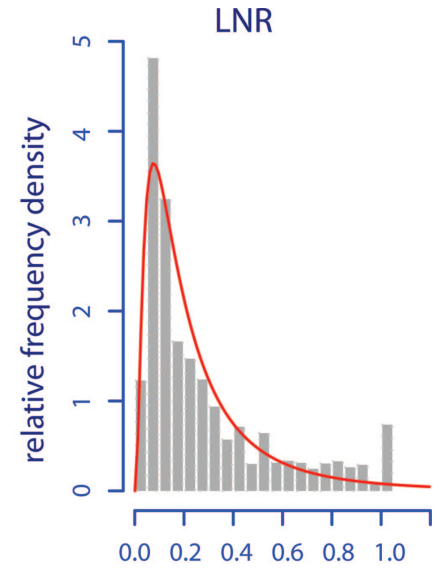

Lymph node ratio

Figure 1. The distribution of the number of positive nodes, the number of examined nodes, and the LNR, without taking into account age. Unadjusted histograms. Red curves are continuous distribution functions fitted by GAMLSS: lognormal for npos, normal for $n t o t$, and lognormal for LNR.

Abbreviations: LNR, lymph node ratio; npos, number of positive nodes; ntot, number of examined nodes.
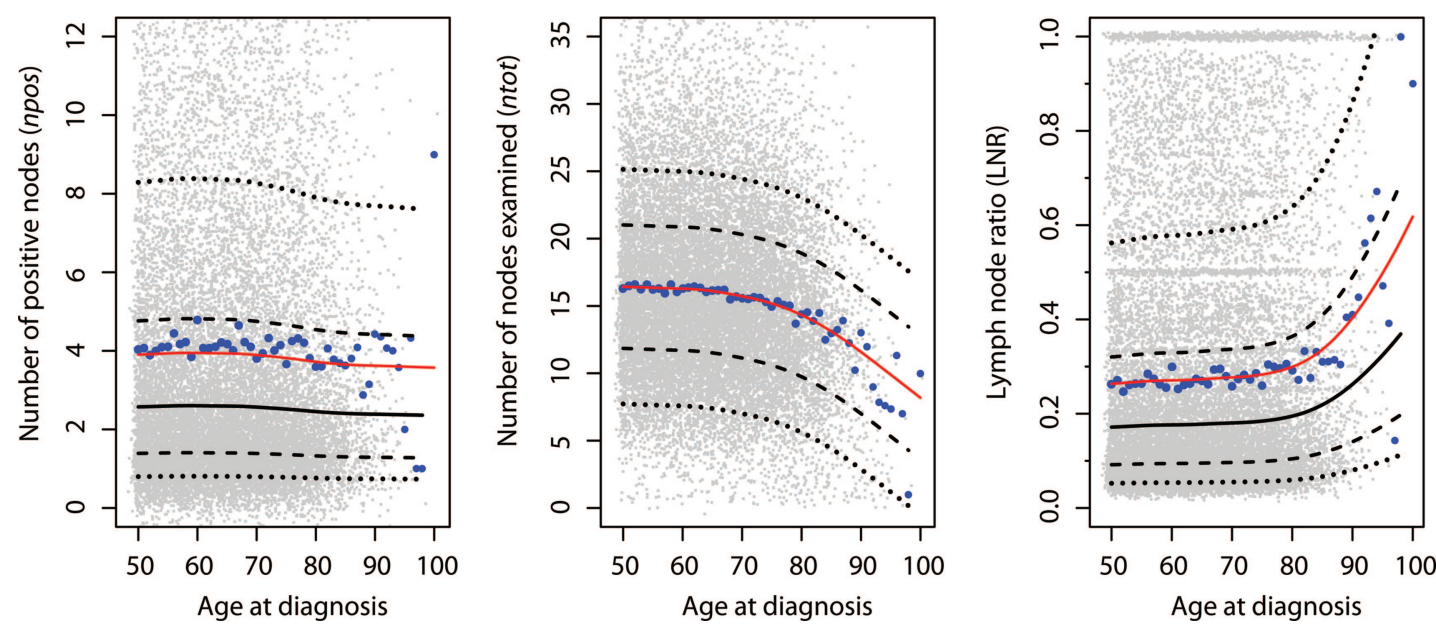

Figure 2. The distribution of the number of positive nodes, the number of nodes examined, and the LNR, as a function of age at diagnosis. The three panels represent the distribution of npos, ntot, and LNR according to age. Two representations are combined in each panel, on one hand crude unadjusted observed data, represented by gray and blue dots, and on the other hand semiparametric smoothing by GAMLSS, represented by continuous curves. The gray dots are jittered individual patients' lymph node values, and the large blue dots are the average lymph node values computed separately at each age. The curves are respectively the mean (plain curve in red), the median (plain curve in black), the 25th and 75th percentile (lower and upper dashed curve in black), and the 10th and 90th percentile (lower and upper dotted curve in black). In the ntot panel, the mean and the median curves are identical.

Abbreviations: LNR, lymph node ratio; npos, number of positive nodes; ntot, number of examined nodes.

lymph node ratio came on top of the variables selected by the BIC, followed by T stage, hormone receptor status, marital status, local therapy, histological grade, and race (Table 3). The number of positive nodes was also selected by the BIC. It ranked 9th at the bottom of the list and caused instabilities in subset analyses due to colinearity with LNR; therefore, we rejected it. On the basis of the retained variables, the cause-specific hazard ratios in Table 3 showed that age was strongly associated with mortality from other causes, whereas LNR was preponderantly associated with mortality from breast cancer. Other variables showing a differential association with mortality from breast cancer were $\mathrm{T}$ stage, hormone receptor status, and histological grade. Marital status, local therapy, and race showed less differential association with the cause-specific endpoints.

Last, the models of Table 3 were recomputed by taking into account interactions between the age and LNR categories. Hazard ratios of the other covariates were practically unaffected; therefore, Table 4 summarizes only the results pertaining to age and LNR. Combined age and LNR were 

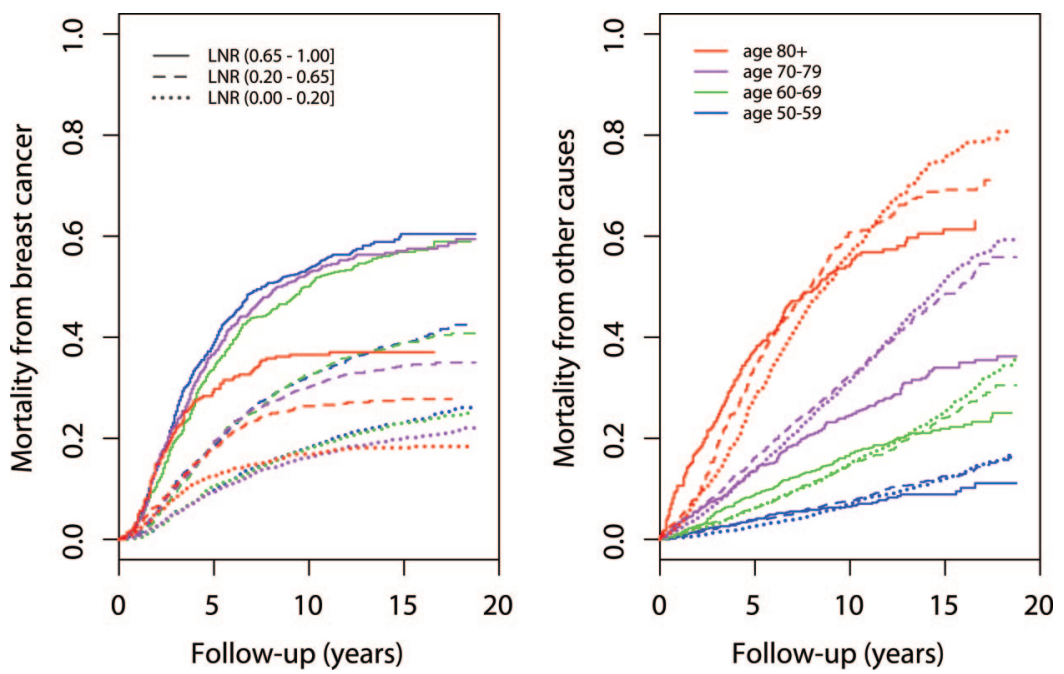

Figure 3. Cause-specific mortality according to age and LNR. The two panels represent the estimated cause-specific probabilities of dying, either from breast cancer (A) or from other causes (B). The curves are coded by type to indicate the LNR group (plain $=$ high-risk LNR; dashed = intermediary-risk LNR; dotted $=$ low-risk LNR) and by color to indicate the age group (red $=$ age $\geq 80$ years, purple $=70-79$ years, green $=60-69$ years, and blue $=50-59$ years). The same coding applies to the two panels, for example, the red plain curves represent age $\geq 80$ years with high LNR and the purple dashed curves represent age 70-79 years with intermediary LNR. (A): Apart a late deviation of the plain red curve, all curves of the same type are clustered together irrespective of their colors, indicating that patients with the same LNR have comparable risks of dying from breast cancer, regardless of age. (B): Curves of the same color are clustered together irrespective of their types, indicating that patients in the same age group have comparable risks of dying from other causes, regardless of LNR values. The overall mortality can be computed from the two panels by adding the cause-specific mortalities. For example, age 50-59 years with high-risk LNR is represented by the plain blue curves. At 5 years the corresponding breast cancer mortality reads 0.39 , the other causes mortality reads 0.04 , and the resulting 5-year overall risk of dying is 0.43 (see also Tables $2 \mathrm{~A}$ and $2 \mathrm{~B}$ ).

Abbreviation: LNR, lymph node ratio.

associated with an increasing overall relative mortality up to sevenfold, with a hazard ratio of 7.49 [6.54-8.59] in women $\geq 80$ years with a high LNR, as compared with women 50-59 years old with a low LNR.

\section{DISCUSSION}

The literature has investigated issues of lymph nodes and age in breast cancer, but to the best of our knowledge, not how the extent of nodal involvement changes with age and how this change affects survival. Wildiers et al. looked at the incidence of positive nodal status but did not analyze nodal involvement nor the impact on survival [37]. Schonberg et al. investigated the impact of age but not that of the extent of nodal involvement [38]. We have investigated many facets of nodal involvement [39-43]; we have investigated the functional effect of age and survival [44], but like other authors, not how age and nodes relate, and foremost, until now, not how that relationship jointly affects survival. Moreover, recently Danko et al. validated the LNR applied to the Duke University Medical Center breast cancer tumor registry [45]. Greene intervened in the discussion as Editor of the current AJCC [46]. In consideration of the LNR for inclusion in the future 8th edition of the TNM Staging Strategy, one of the questions Greene raised was "because the total number of lymph nodes is reduced as we get older, what is the value of the lymph node ratio in the staging of older patients with cancer?" [45]. We believe that the present study is a timely key contribution.

Our methods warrant some comments. Describing and displaying the relationship between age and lymph nodes is a nontrivial issue. We wanted to convey a picture of the trends, but we also wanted to convey a direct visualization of the data "as is," its strengths as well as its weaknesses. Figure 1 showed histograms of lymph node values without taking into account age. Extending the figure to take into account age would have required the stacking of 50 histograms, one for each year of age, which would have been visually unacceptable. Stacking fewer histograms, or using summary box plots, would have lost fine details. We designed Figure 2 by combining several tools: jittering applied to each and every patients' data to display its heterogeneity [47], and ordinary averages to display the crude trends, on which we superimposed smoothed modeling based on GAMLSS to display a summary of the patterns.

GAMLSS is a new set of tools that allow all the parameters (e.g., the mean) of the distribution (e.g., the lognormal) of a response variable (e.g., the LNR) to be modeled as 
Table 1. Patient characteristics according to age

\begin{tabular}{|c|c|c|c|c|c|}
\hline & $\begin{array}{c}\text { All ages } \\
(n=17685)\end{array}$ & $\begin{array}{c}\text { Age 50-59 yr(s) } \\
(n=5863)\end{array}$ & $\begin{array}{c}\text { Age 60-69 yr(s) } \\
(n=5716)\end{array}$ & $\begin{array}{l}\text { Age } 70-79 \text { yr(s) } \\
(n=4431)\end{array}$ & $\begin{array}{c}\text { Age } \geq 80 \text { yr(s) } \\
(n=1675)\end{array}$ \\
\hline & $n$ & $\%$ & $\%$ & $\%$ & $\%$ \\
\hline Overall & 17685 & 33.2 & 32.3 & 25.1 & 9.5 \\
\hline \multicolumn{6}{|l|}{ Registry area } \\
\hline Eastern (CT, Detroit, Atlanta) & 7072 & 33.5 & 32.7 & 25.1 & 8.7 \\
\hline Central (IA, NM, UT) & 4364 & 30.3 & 31.3 & 26.4 & 12.0 \\
\hline $\begin{array}{l}\text { Western (San Francisco, } \\
\text { Hawaii, Seattle) }\end{array}$ & 6249 & 34.8 & 32.6 & 24.1 & 8.5 \\
\hline \multicolumn{6}{|l|}{ Year of diagnosis } \\
\hline 1988-1992 & 8731 & 30.3 & 35.0 & 25.2 & 9.6 \\
\hline 1993-1997 & 8954 & 36.0 & 29.8 & 24.9 & 9.3 \\
\hline Married status & 9990 & 40.3 & 35.5 & 20.5 & 3.6 \\
\hline Black race & 1319 & 39.8 & 32.4 & 22.4 & 5.5 \\
\hline Tumor medial location & 1896 & 35.8 & 33.0 & 23.8 & 7.4 \\
\hline Ductal histology & 13985 & 33.5 & 32.2 & 24.6 & 9.6 \\
\hline \multicolumn{6}{|l|}{ Histological grade } \\
\hline Grade $1-2$ & 6523 & 32.8 & 31.2 & 26.0 & 9.9 \\
\hline Grade 3-4 & 6231 & 35.9 & 31.6 & 23.8 & 8.7 \\
\hline Unknown & 4931 & 30.1 & 34.7 & 25.3 & 9.9 \\
\hline \multicolumn{6}{|l|}{ Hormone receptor status } \\
\hline $\mathrm{ER}+\mathrm{PR}+$ & 7714 & 32.3 & 31.7 & 26.5 & 9.6 \\
\hline $\mathrm{ER}-\mathrm{PR}-$ & 2211 & 41.8 & 32.7 & 19.1 & 6.4 \\
\hline $\mathrm{ER}+\mathrm{PR}-$ & 1620 & 32.6 & 31.6 & 25.3 & 10.5 \\
\hline $\mathrm{ER}-\mathrm{PR}+$ & 350 & 45.4 & 29.1 & 18.0 & 7.4 \\
\hline ER and/or PR unknown & 5790 & 30.4 & 33.5 & 25.8 & 10.3 \\
\hline \multicolumn{6}{|l|}{ Local therapy } \\
\hline Mastectomy only & 11150 & 28.4 & 31.9 & 27.6 & 12.0 \\
\hline Mastectomy plus RT & 1755 & 39.1 & 33.2 & 22.8 & 4.8 \\
\hline BCS only & 1075 & 44.9 & 29.8 & 14.9 & 10.4 \\
\hline BCS plus RT & 3705 & 41.1 & 34.0 & 21.3 & 3.6 \\
\hline T2 stage (tumor size $>2 \mathrm{~cm}$ ) & 8558 & 33.3 & 31.3 & 24.6 & 10.9 \\
\hline \multicolumn{6}{|l|}{ No. nodes examined } \\
\hline $1-9$ & 2924 & 27.9 & 29.1 & 27.2 & 15.8 \\
\hline$\geq 10$ & 14761 & 34.2 & 33.0 & 24.6 & 8.2 \\
\hline \multicolumn{6}{|l|}{ No. positive nodes } \\
\hline $1-3$ & 11661 & 33.0 & 32.0 & 25.2 & 9.9 \\
\hline $4-9$ & 4064 & 34.1 & 32.0 & 24.7 & 9.2 \\
\hline$\geq 10$ & 1960 & 32.1 & 35.1 & 25.1 & 7.8 \\
\hline \multicolumn{6}{|l|}{ Lymph node ratio } \\
\hline $0.01-0.20$ & 10205 & 33.9 & 32.6 & 24.7 & 8.8 \\
\hline $0.21-0.65$ & 5242 & 33.4 & 31.8 & 25.4 & 9.5 \\
\hline $0.66-1.00$ & 2238 & 29.1 & 32.4 & 25.9 & 12.6 \\
\hline \multicolumn{6}{|l|}{ Cause of death } \\
\hline Alive & 7563 & 46.0 & 35.1 & 17.0 & 1.8 \\
\hline Breast cancer & 5153 & 34.3 & 33.8 & 24.1 & 7.8 \\
\hline Other causes & 4969 & 12.4 & 26.5 & 38.3 & 22.8 \\
\hline
\end{tabular}

Figures in the last four columns are row percentages.

Abbreviations: BCS, breast-conserving surgery; ER, estrogen receptor; PR, progesterone receptor; RT, radiation therapy. 
Table 2A. Cause-specific mortality at 5 years, according to age and to LNR

\begin{tabular}{|c|c|c|c|c|c|}
\hline & Age 50-59 yr(s) & Age 60-69 yr(s) & Age 70-79 yr(s) & Age $\geq 80$ yr(s) & All ages \\
\hline \multicolumn{6}{|c|}{ (a) Breast cancer mortality at 5 years } \\
\hline Low LNR & 9.8 & 10.5 & 9.4 & 12.6 & 10.2 \\
\hline Mid-LNR & 18.6 & 19.2 & 19.5 & 18.1 & 19.0 \\
\hline High LNR & 39.0 & 34.1 & 36.9 & 29.8 & 35.7 \\
\hline All LNR & 15.7 & 16.0 & 16.0 & 17.1 & 16.0 \\
\hline \multicolumn{6}{|c|}{ (b) Other causes mortality at 5 years } \\
\hline Low LNR & 2.6 & 6.3 & 14.0 & 28.2 & 8.9 \\
\hline Mid-LNR & 3.8 & 6.2 & 16.2 & 34.2 & 10.6 \\
\hline High LNR & 4.0 & 9.0 & 13.5 & 37.6 & 12.3 \\
\hline All LNR & 3.1 & 6.6 & 14.6 & 31.5 & 9.8 \\
\hline \multicolumn{6}{|c|}{ (c) All causes mortality at 5 years } \\
\hline Low LNR & 12.5 & 16.7 & 23.5 & 40.8 & 19.1 \\
\hline Mid-LNR & 22.5 & 25.4 & 35.7 & 52.3 & 29.6 \\
\hline High LNR & 43.0 & 43.1 & 50.3 & 67.4 & 48.0 \\
\hline All LNR & 18.8 & 22.6 & 30.7 & 48.7 & 25.9 \\
\hline
\end{tabular}

Estimated cause-specific risk of dying at 5 years (\%), from breast cancer (a) and from other causes (b). The last column and last row of each section show the risks computed without taking into account age and LNR, respectively. (a): Italicized column: The risk of dying from breast cancer increases with LNR, similarly to the other columns. (b): Italicized row: The risk of dying from other causes increases with age, similarly to the other rows. (c): The overall risk of dying increases with both LNR and age. Low LNR $=0.01-0.20$; mid-LNR $=0.21-0.65$; high LNR $=0.66-1.00$.

Abbreviation: LNR, lymph node ratio.

Table 2B. $95 \%$ confidence intervals of the 5-years cause-specific mortalities

\begin{tabular}{|c|c|c|c|c|c|}
\hline & Age 50-59 yr(s) & Age 60-69 yr(s) & Age 70-79 yr(s) & Age $\geq 80$ yr(s) & All ages \\
\hline \multicolumn{6}{|c|}{ (a) Breast cancer mortality at 5 yr(s): $95 \%$ CI } \\
\hline Low LNR & $8.8-10.8$ & $9.4-11.5$ & $8.2-10.7$ & $10.1-15.0$ & $9.6-10.8$ \\
\hline Mid-LNR & $16.7-20.5$ & $17.3-21.2$ & $17.1-21.8$ & $13.9-22.1$ & 17.9-20.1 \\
\hline High LNR & $35.1-42.7$ & $30.4-37.6$ & $32.5-40.9$ & $22.7-36.1$ & $33.6-37.8$ \\
\hline All LNR & $14.7-16.6$ & $15.0-17.0$ & $14.9-17.2$ & $15.0-19.2$ & $15.5-16.6$ \\
\hline \multicolumn{6}{|c|}{ (b) Other causes mortality at 5 yr(s): $95 \%$ CI } \\
\hline Low LNR & $2.1-3.2$ & $5.4-7.1$ & $12.6-15.5$ & $24.9-31.3$ & $8.3-9.5$ \\
\hline Mid-LNR & $2.8-4.8$ & $4.9-7.5$ & $14.0-18.4$ & $29.4-38.6$ & $9.7-11.5$ \\
\hline High LNR & $2.0-5.9$ & $6.4-11.5$ & $9.9-16.9$ & $30.4-43.9$ & $10.6-14.0$ \\
\hline All LNR & $2.7-3.6$ & $5.9-7.3$ & $13.5-15.8$ & $29.0-34.0$ & $9.3-10.3$ \\
\hline \multicolumn{6}{|c|}{ (c) All causes mortality at $5 \mathrm{yr}(\mathrm{s}): 95 \% \mathrm{CI}$} \\
\hline Low LNR & $11.4-13.6$ & $15.5-18.0$ & $21.8-25.1$ & $37.5-43.9$ & $18.3-19.8$ \\
\hline Mid-LNR & $20.5-24.4$ & $23.3-27.5$ & $33.1-38.2$ & $47.7-56.5$ & $28.4-30.8$ \\
\hline High LNR & $39.1-46.7$ & $39.4-46.6$ & $46.1-54.3$ & $61.4-72.4$ & $45.9-50.0$ \\
\hline All LNR & $17.8-19.8$ & $21.5-23.7$ & $29.3-32.0$ & $46.2-51.0$ & $25.2-26.5$ \\
\hline
\end{tabular}

linear/nonlinear or smooth functions (e.g., cubic spline) of the explanatory variables (e.g., age) [35]. The GAMLSS has been selected by the World Health Organization to establish standardized growth curves [48, 49]. Khondoker et al. found the GAMLSS to be a more powerful method than other variance stabilizing methods for the normalization of cDNA microarray data [50]. Mouksassi et al. used the GAMLSS to simulate age-matched weight data in a pediat- 
Table 3. Competing risks analysis

\begin{tabular}{|c|c|c|c|c|c|c|c|}
\hline \multirow[b]{2}{*}{ Variable } & \multirow[b]{2}{*}{ Reference } & \multicolumn{2}{|c|}{ Breast cancer } & \multicolumn{2}{|c|}{ Other causes } & \multicolumn{2}{|c|}{ Overall } \\
\hline & & $\mathbf{H R}_{\mathrm{BC}}$ & $95 \% \mathrm{CI}$ & HR $_{\text {OC }}$ & $95 \% \mathrm{CI}$ & HR $_{\text {OV }}$ & $95 \% \mathrm{CI}$ \\
\hline \multicolumn{8}{|l|}{ Age at diagnosis } \\
\hline $60-69$ yr(s) & Age 50-59 & 1.04 & $(0.97-1.11)$ & 2.23 & $(2.03-2.46)$ & 1.35 & $(1.28-1.43)$ \\
\hline $70-79$ yr(s) & Age 50-59 & 1.12 & $(1.04-1.20)$ & 5.18 & $(4.72-5.68)$ & 2.15 & $(2.04-2.27)$ \\
\hline$\geq 80$ yr(s) & Age 50-59 & 1.15 & $(1.03-1.29)$ & 11.92 & $(10.7-13.2)$ & 3.67 & $(3.43-3.93)$ \\
\hline \multicolumn{8}{|l|}{ Lymph node ratio } \\
\hline $0.21-0.65$ & $0.01-0.20$ & 1.82 & $(1.71-1.94)$ & 1.18 & $(1.11-1.26)$ & 1.45 & $(1.38-1.51)$ \\
\hline $0.66-1.00$ & $0.01-0.20$ & 3.41 & $(3.16-3.67)$ & 1.34 & $(1.22-1.47)$ & 2.25 & $(2.13-2.38)$ \\
\hline T2 stage & $\mathrm{T} 1($ size $\leq 2 \mathrm{~cm})$ & 1.68 & $(1.58-1.78)$ & 1.17 & $(1.11-1.24)$ & 1.39 & $(1.34-1.45)$ \\
\hline \multicolumn{8}{|l|}{ Hormone receptor } \\
\hline $\mathrm{ER}+\mathrm{PR}+$ & ER PR $+/-,-/+$, unknown & 0.76 & $(0.71-0.81)$ & 0.93 & $(0.87-0.98)$ & 0.84 & $(0.81-0.88)$ \\
\hline $\mathrm{ER}-\mathrm{PR}-$ & ERPR $+/-,-/+$, unknown & 1.30 & $(1.20-1.41)$ & 1.01 & $(0.90-1.12)$ & 1.21 & $(1.14-1.29)$ \\
\hline Married status & Nonmarried, unknown & 0.87 & $(0.82-0.92)$ & 0.72 & $(0.68-0.77)$ & 0.79 & $(0.76-0.83)$ \\
\hline \multicolumn{8}{|l|}{ Local therapy } \\
\hline Mastectomy plus RT & Mastectomy only & 0.88 & $(0.81-0.96)$ & 0.91 & $(0.82-1.01)$ & 0.91 & $(0.85-0.98)$ \\
\hline BCS only & Mastectomy only & 0.79 & $(0.69-0.89)$ & 0.94 & $(0.83-1.07)$ & 0.85 & $(0.78-0.94)$ \\
\hline BCS plus RT & Mastectomy only & 0.73 & $(0.68-0.79)$ & 0.80 & $(0.74-0.87)$ & 0.76 & $(0.72-0.81)$ \\
\hline Histological grade $3-4$ & Grade $1-2$, unknown & 1.41 & $(1.33-1.49)$ & 0.99 & $(0.93-1.06)$ & 1.19 & $(1.15-1.25)$ \\
\hline Black race & Other, unknown & 1.33 & $(1.21-1.46)$ & 1.29 & $(1.16-1.44)$ & 1.31 & $(1.22-1.41)$ \\
\hline \multicolumn{8}{|c|}{$\begin{array}{l}\mathrm{HR} \text { and } 95 \% \mathrm{CI} \text {, computed for specific } \mathrm{BC} \text { and } \mathrm{OC} \text { mortality and for overall mortality. The variables were ranked according } \\
\text { to the Bayesian Information Criterion. } \mathrm{HRs} \text { of variables with a large differential effect on a specific mortality are italicized. } \\
\text { Note that the overall mortality hazard ratios } \mathrm{HR}_{\mathrm{OV}} \text { can be approximated from the } \mathrm{BC} \text { and OC hazard ratios as } \\
\left.\left.\left.\text { exp[(log(HR} \mathrm{HC}_{\mathrm{BC}}\right)+\log \left(\mathrm{HR}_{\mathrm{OC}}\right)\right) / 2\right] \text {. } \\
\text { Abbreviations: } \mathrm{BC} \text {, breast cancer; BCS, breast-conserving surgery; CI, confidence interval; ER, estrogen receptor; HR, } \\
\text { hazard ratio; OC, other causes; PR, progesterone receptor; RT, radiation therapy. }\end{array}$} \\
\hline
\end{tabular}

Table 4. Adjusted joint effect of age and LNR on mortality

\begin{tabular}{|c|c|c|c|c|c|c|c|c|}
\hline & \multicolumn{2}{|c|}{ Age 50-59 yr(s) } & \multicolumn{2}{|c|}{ Age 60-69 yr(s) } & \multicolumn{2}{|c|}{ Age 70-79 yr(s) } & \multicolumn{2}{|c|}{ Age $\geq 80$ yr(s) } \\
\hline & HR & $95 \% \mathrm{CI}$ & HR & $95 \% \mathrm{CI}$ & HR & $95 \% \mathrm{CI}$ & HR & $95 \% \mathrm{CI}$ \\
\hline \multicolumn{9}{|c|}{ (a) Breast cancer mortality } \\
\hline Low LNR & 1 & & 1.07 & $(0.97-1.19)$ & 1.04 & $(0.93-1.17)$ & 1.24 & $(1.04-1.47)$ \\
\hline Mid-LNR & 1.82 & $(1.64-2.02)$ & 1.89 & $(1.70-2.10)$ & 2.04 & $(1.81-2.30)$ & 2.08 & $(1.72-2.50)$ \\
\hline High LNR & 3.41 & $(3.00-3.87)$ & 3.36 & $(2.97-3.80)$ & 4.21 & $(3.69-4.81)$ & 3.56 & $(2.89-4.39)$ \\
\hline \multicolumn{9}{|c|}{ (b) Other causes mortality } \\
\hline Low LNR & 1 & & 2.29 & $(2.02-2.59)$ & 5.26 & $(4.67-5.93)$ & 11.87 & $(10.39-13.56)$ \\
\hline Mid-LNR & 1.22 & $(1.03-1.46)$ & 2.51 & $(2.17-2.90)$ & 6.42 & $(5.63-7.33)$ & 14.14 & $(12.13-16.48)$ \\
\hline High LNR & 1.35 & $(1.02-1.79)$ & 3.31 & $(2.73-4.00)$ & 6.17 & $(5.15-7.39)$ & 17.55 & $(14.53-21.20)$ \\
\hline \multicolumn{9}{|c|}{ (c) All causes mortality } \\
\hline Low LNR & 1 & & 1.48 & $(1.37-1.59)$ & 2.41 & $(2.23-2.61)$ & 4.46 & $(4.06-4.90)$ \\
\hline Mid-LNR & 1.66 & $(1.52-1.82)$ & 2.12 & $(1.94-2.31)$ & 3.42 & $(3.14-3.73)$ & 5.54 & $(4.96-6.19)$ \\
\hline High LNR & 2.97 & $(2.65-3.32)$ & 3.49 & $(3.14-3.87)$ & 4.96 & $(4.45-5.52)$ & 7.49 & $(6.54-8.59)$ \\
\hline
\end{tabular}


ric short-bowel syndrome population to optimize phase I therapeutic dosing strategies [51]. Though few, these studies show the emerging importance of the GAMLSS. The present study is among the first large-scale clinical applications of the GAMLSS.

By the book, our GAMLSS choices of lognormal and normal distributions for the numbers nodes might be considered inappropriate. The lognormal and normal distributions apply to continuous data but not to discrete data [43]. Given that numbers of nodes are counted as integers, a median of, for example, "3.721 positive nodes" is incorrect. We applied discrete distributions such as the negative binomial that we had previously investigated [43]. Surprisingly, the percentiles did not match the data any better, with the additional drawback of sudden jumps when displaying percentile curves, for example, a median of 2 nodes at age 69 years, which abruptly became 3 nodes at age 70 years. We found that the continuous distributions not only obviated the sudden jumps but also biologically were a more sensible choice. On reflection, integer units of lymph nodes are artificial abstractions. Uninvolved lymph nodes can widely differ within a patient and among patients, and their involvement can be minimal, or can be any range through to massive involvement or even beyond the node [52] —yet all of these cases are lumped together by coarse integer units. We have argued that breast cancer is a disease continuum without identifiable cutpoints [53]. By modeling the numbers of nodes as continuous, stating a median of " 3.721 positive nodes," though perplexing, is in fact closer to the reality of the disease than stating "4 nodes."

Other than tongue-in-cheek procedures names, there is no magic recipe for the selection of variables in multivariate analyses. Investigators have to choose among many selection methods, automatic and/or handpicked, and among diverse criteria for selection. We have favored the use of the Akaike Information Criterion (AIC) in our previous studies where the aim was to detect subtle differences or where modeling was applied to small data sets [33, 54-56]. The AIC penalizes models by the number of parameters included in the models: the more variables included, the higher the penalty. In the present study, we strived for a model that should be as parsimonious as possible, that is, more severe than the AIC, but not to the extreme of discarding all variables. We chose the BIC on consideration of the extensive literature [57-60]. The BIC penalizes models by the logarithm of total number of cases, multiplied by the number of parameters: the more variables included, and the more cases there are, the higher the penalty. We applied a semiautomatic method to reduce investigator's influence, using a programming procedure for which there is longstanding support [36]. As shown in Table 3, pending a min- imal manual pruning that we did for npos, the model was reasonably lean, yet was capable of retaining variables known to be important in breast cancer.

Our results are biologically consistent. Table 3 shows that estrogen receptor positive progesterone receptor positive $(\mathrm{ER}+\mathrm{PR}+)$ was associated with reduced risk of $\mathrm{BC}$ death and $\mathrm{ER}-\mathrm{PR}$ - with increased risk of BC death, but were not related to "Other causes" of death. Likewise, high grade and T stage were associated with $\mathrm{BC}$ death. Note that the differential effects were not clear-cut, as LNR and T2 stage were also associated with some increased risk of other causes of death. Potential explanations are misclassification of cause of death or lead-time bias in the diagnosis of breast cancer in nonscreened patients. Race was associated with both increased risk of $\mathrm{BC}$ death and increased risk of other causes of death. Breast-conserving surgery was associated with a reduced risk of $\mathrm{BC}$ death, possibly because of selection of patients with more favorable characteristics such as tumors diagnosed by screening or resected with wide clear margins or other factors that the present analyses could not account for.

It is known that the incidence of node-positive status decreases with age, as a consequence of the smaller proportion of patients receiving any axillary lymph node dissection, which has also been reported to decrease with age [22, 61]. However, elderly people diagnosed with breast cancer were significantly more likely to die from cancer, even with less aggressive tumors. This may be due to inadequate treatment or other unknown factors [14, 62]. Wildiers et al. found that lymph node involvement probably decreased until 70 years but increased again thereafter [37]. We found that npos did not change notably over the age range 50-90 years, whereas $n$ tot declined with age (Figure 2). In the present data, an unchanged npos in older women masked the disease severity because of the reduced number of lymph node examinations, suggesting understaging in keeping with other authors who noted that axillary examinations might have been performed less in elderly patients [25].

Similarly to other authors, we found that increased age was associated with less aggressive tumor characteristics $[7,8,10-13]$ : the relative proportion of $\mathrm{ER}+\mathrm{PR}+$ over $\mathrm{ER}-\mathrm{PR}-$, and the relative proportion of low grade over high grade, increased with age (Table 1). However, results of our survival analyses do not concur with tumor indolency. Table 1 shows that the unadjusted risk of dying from breast cancer increased with age: the relative proportion of patients dying of breast cancer over those still living increased from $34.3 \% / 46.0 \%$ at $50-59$ years of age to $7.8 \%$ / $1.8 \%$ at $\geq 80$ years of age, although this was overshadowed by a marked increase of non-breast cancer deaths, from 
$12.4 \% / 46.0 \%$ to $22.8 \% / 1.8 \%$ (Table 1 ). This parallels previous findings that disease-free survival was not associated with age [63]. In the adjusted multivariate analysis, assuming all other factors equal, age was not associated with a reduction of breast cancer death, specific hazard ratio of 1.04 at age 60-69 years and 1.15 at age $\geq 80$ years (Table 3 ). Our analyses did not investigate the changes of therapy with age. Nevertheless, in view of the high risk of breast cancer death associated with high tumor burden (Table 4), we cannot exclude the possibility that elderly women with high tumor burdens might have been undertreated [14, 64, 65].

Results of our study concur with previous studies showing an association between lymph node dissection and reduced mortality in other tumors [66]. It has been hypothesized that dissection removes a potential route of metastasis, resulting in improved survival [61]. Lower use of axillary dissection in elderly women may be related to this proposed phenomenon.

Besides addressing the value of the LNR in the staging of older patients [45], our results have important clinical implications because of the aging of populations in developed countries, and in particular the ones aged 65 or older. In the United States, it is estimated that 36.8 million (12\%) of the U.S. population are 65 years and older and this number is expected to double in the next 2 decades with an equal proportion of men and women [67]. In contrast to previous generations, baby boomers tend to live longer and have a healthier lifestyle. As an illustration, the life expectancy of individuals aged $65-74,75-84$, and 85 years is estimated to be 15,10 , and 6 years, respectively [68]. Traditionally, older adults are less likely to receive optimal doses of chemotherapy compared with younger patients because of the presence of comorbidities and increased risk of complications $[64,69,70]$. However, in selected patients with positive axillary lymph nodes and estrogen receptor negative tumors, adjuvant chemotherapy may improve outcome [71]. In a retrospective analysis of 40,000 elderly breast cancer patients, adjuvant chemotherapy was proven to improve survival in patients $\geq 70$ years, ER negative, and axillary node positive [72]. The benefit of chemotherapy for elderly ER negative breast cancer patients was also corroborated in another study [73]. Thus, this subset of elderly breast cancer patients may benefit from chemotherapy if they have a life expectancy of at least 5 years. Use of LNR as a cutoff for elderly patients may help clinicians guide their treatment decisions. In patients with $L N R \leq 0.20$, observation may be justified following surgery with treatment initiated at the time of recurrence. Chemotherapy and/or radiotherapy may be considered for patients with LNR $>0.20$ and fit physical conditions. In very old patients, the risk of dying from causes other than breast cancer was exceedingly high. Nevertheless, a LNR $>0.65$ identified among the very old patients a risk of dying from breast cancer commensurately high. The high-LNR rows of Table 4 show that, in the oldest age group where the hazard ratio of "Other causes" appeared the highest at 17.55, the hazard ratio of breast cancer death was 3.56, versus an overall hazard ratio of 7.49. This hints that breast cancer represented the single most important cause of death in patients with LNR $>0.65$. Actually, we did not detail the "Other causes" of death. Counting the causes of death in the $\geq 80$ years age group with LNR $>0.65$, we found the following: 13 patients were still living or were assumed so and 104 patients died from breast cancer, 96 from cardiovascular causes, 12 from respiratory diseases, 13 from other cancers, and 43 from diverse other causes.

Limitations of the study include its retrospective nature. Data were collected at the time of breast cancer diagnosis, thereby eliminating recall bias. However, information on systemic therapy was not collected. Modeling could not take into account whether patients received hormone therapy or neoadjuvant chemotherapy which could affect lymph node values [74]. We cannot exclude that the effect of age and lymph node ratio on breast cancer mortality might have been biased by the discrepancy between adjuvant treatment (chemo- and radiotherapy) received by the different age groups. Additionally, comorbidity is increasingly important with advancing age, which might confound results [75]. As shown in Figure 3 and Tables $1-4$, age is a strong predictor of non-breast cancer death. Data on comorbidities are not collected by the SEER registries.

Additional limitations included analyses restricted to women who underwent axillary exploration and for whom more complete pathological information was available. We cannot exclude the potential bias of missing data by excluding patients without lymph nodes examined. A note of caution is also warranted, considering that a ratio based on a small number of lymph nodes has a larger standard error; this could affect the reliability of the lymph node ratio in the older age group who had less extensive axillary dissection.

Despite these limitations, the study has several strengths supporting its validity and reliability. Since its establishment in 1973, the SEER has considerably expanded, providing valuable material to build research hypotheses and to gain insight into populations under-represented in clinical trials $[38,76]$. SEER data are derived from populationbased cancer registries, which minimize or eliminate several study biases and confounders, including selection biases and treatment fads. SEER data have been shown to be nearly complete, reducing the influence of loss to fol- 
low-up biases. Highly accurate data collection at the time of diagnosis reduces recall biases. Overall, we believe that the data analyzed are well balanced; implications of our results strongly suggest tumor burden, as measured by the LNR, should be taken into account when evaluating treatment decisions in elderly patients.

\section{CONCLUSIONS}

Age at diagnosis was nonlinearly related to the lymph node ratio, indicating a greater burden of disease with advancing age. Elderly women with a higher tumor burden had an increased risk of breast cancer death added on top of other risks. We argue that tumor burden measured by LNR should be taken into account in order to advise optimal breast cancer therapy in elderly patients.

\section{ACKNOWLEDGMENTS}

We gratefully acknowledge an anonymous reviewer who opened the way to a trailblazing revision of the manuscript.

\section{Author Contributions}

Conception/Design: Vincent Vinh-Hung, Sue A. Joseph

Collection and/or assembly of data: Vincent Vinh-Hung

Data analysis and interpretation: Vincent Vinh-Hung, Sue A. Joseph, Nadege Coutty, Bevan Hong Ly, Georges Vlastos, Nam Phong Nguyen

Manuscript writing: Vincent Vinh-Hung, Sue A. Joseph, Nadege Coutty, Bevan Hong Ly, Georges Vlastos, Nam Phong Nguyen

Final approval of manuscript: Vincent Vinh-Hung, Sue A. Joseph, Nadege Coutty, Bevan Hong Ly, Georges Vlastos, Nam Phong Nguyen

\section{REFERENCES}

1 Boivin-Angele S, Hall J, Sasco AJ et al. Breast Cancer. Lyon, France: IARC Press, 2003:188-193. Available at http://www.iarc.fr. Accessed January 23, 2010.

2 Jemal A, Ward E, Thun MJ. Recent trends in breast cancer incidence rates by age and tumor characteristics among U.S. women. Breast Cancer Res 2007;9:R28

3 Héry C, Ferlay J, Boniol $\mathrm{M}$ et al. Changes in breast cancer incidence and mortality in middle-aged and elderly women in 28 countries with Caucasian majority populations. Ann Oncol 2008;19:1009-1018.

4 Peto R, Boreham J, Clarke M et al. UK and USA breast cancer deaths down $25 \%$ in year 2000 at ages 20-69 years. Lancet 2000;355(9217):1822.

5 Jatoi I, Chen BE, Anderson WF et al. Breast cancer mortality trends in the United States according to estrogen receptor status and age at diagnosis. J Clin Oncol 2007;25:1683-1690.

6 Hughes S, Barbachano Y, Ashley S et al. Time trends in the outcome of elderly patients with breast cancer. Breast J 2008;14:158-163.

7 Fisher CJ, Egan MK, Smith P et al. Histopathology of breast cancer in relation to age. Br J Cancer 1997;75:593-596.

8 Diab SG, Elledge RM, Clark GM. Tumor characteristics and clinical outcome of elderly women with breast cancer. J Natl Cancer Inst 2000;92: $550-556$.

9 Balducci L. Geriatric oncology. Crit Rev Oncol Hematol 2003;46:211220

10 Daidone MG, Coradini D, Martelli G et al. Primary breast cancer in elderly women: biological profile and relation with clinical outcome. Crit Rev Oncol Hematol 2003;45:313-325.

11 Gennari R, Curigliano G, Rotmensz N et al. Breast carcinoma in elderly women: features of disease presentation, choice of local and systemic treatments compared with younger postmenopasual patients. Cancer 2004;101: 1302-1310.

12 Molino A, Giovannini M, Auriemma A et al. Pathological, biological and clinical characteristics, and surgical management, of elderly women with breast cancer. Crit Rev Oncol Hematol 2006;59:226-233.

13 Cheung KL, Wong AW, Parker H et al. Pathological features of primary breast cancer in the elderly based on needle core biopsies-a large series from a single centre. Crit Rev Oncol Hematol 2008;67:263-267.

14 Bouchardy C, Rapiti E, Fioretta G et al. Undertreatment strongly decreases prognosis of breast cancer in elderly women. J Clin Oncol 2003;21:35803587 .
15 Macaskill EJ, Renshaw L, Dixon JM. Neoadjuvant use of hormonal therapy in elderly patients with early or locally advanced hormone receptor-positive breast cancer. The Oncologist 2006;11:1081-1088.

16 Wildiers H, Kunkler I, Biganzoli L et al. Management of breast cancer in elderly individuals: recommendations of the International Society of Geriatric Oncology. Lancet Oncol 2007;8:1101-1115.

17 Balducci L. Treating elderly patients with hormone sensitive breast cancer: what do the data show? Cancer Treat Rev 2009;35:47-56.

18 Hurria A, Wong FL, Pal S et al. Perspectives and attitudes on the use of adjuvant chemotherapy and trastuzumab in older adults with HER-2+ breast cancer: a survey of oncologists. The Oncologist 2009;14:883-890.

19 Woodward WA, Vinh-Hung V, Ueno NT et al. Prognostic value of nodal ratios in node-positive breast cancer. J Clin Oncol 2006;24:2910-2916.

20 Vinh-Hung V, Nguyen NP, Cserni G et al. Prognostic value of nodal ratios in node-positive breast cancer: a compiled update. Future Oncol 2009;5: 1585-1603

21 Joslyn SA, Vinh-Hung V. Correlation of the lymph node ratio with age at diagnosis in post-menopausal women with T1-T2 node positive breast cancer. Abstract 2025. Breast Cancer Res Treat 2006;100:S88.

22 Du X, Freeman JL, Goodwin JS. The declining use of axillary dissection in patients with early stage breast cancer. Breast Cancer Res Treat 1999;53: 137-144.

23 Eaker S, Dickman PW, Bergkvist L et al. Differences in management of older women influence breast cancer survival: results from a populationbased database in Sweden. PLoS Med 2006;3:e25.

24 Halpern MT, Chen AY, Marlow NS et al. Disparities in receipt of lymph node biopsy among early-stage female breast cancer patients. Ann Surg Oncol 2009; 16:562-570.

25 Voogd AC, Nieuwenhuijzen GA, Coebergh JW. Age-related variations in the use of axillary staging may explain the increased risk of axillary lymph node involvement in older women with breast cancer. J Clin Oncol 2009; 27:e276-e277.

26 Greene FL, Page DL, Fleming ID et al. AJCC Cancer Staging Handbook, 6th Edition. New York: Springer Verlag, 2002:255-281.

27 Sobin LH, Wittekind Ch. Breast tumours. In: Sobin LH, Wittekind Ch, eds. TNM Classification of Malignant Tumours, 6th Edition. New-York: Wiley, 2002:131-141.

28 National Cancer Institute. Surveillance, Epidemiology, and End Results (SEER) Program (www.seer.cancer.gov) Public-Use Data (1973-2006), National Cancer Institute, DCCPS, Surveillance Research Program, Cancer 
Statistics Branch, released April 2009, based on the November 2008 submission. Bethesda, MD: National Cancer Institute, 2009. Available at http:// seer.cancer.gov. Accessed April 29, 2009.

29 Rigby RA, Stasinopoulos DM. Generalized additive models for location, scale and shape. Appl Statist 2005;54:507-554.

30 Therneau TM, Grambsch PM. Modeling survival data: extending the Cox model. New York: Springer-Verlag, 2000:87-152.

31 Lunn M, McNeil D. Applying Cox regression to competing risks. Biometrics 1995;51:524-532.

32 Schwarz G. Estimating the dimension of a model. Ann Statist 1978;6:461464.

33 Vinh-Hung V, Verkooijen HM, Fioretta G et al. Lymph node ratio as an alternative to $\mathrm{pN}$ staging in node-positive breast cancer. J Clin Oncol 2009; 27:1062-1068.

34 The R Foundation for Statistical Computing. The R project for statistical computing. The Comprehensive R Archive Network (CRAN) 2009. Available at http://www.r-project.org/. Accessed December 28, 2009.

35 Stasinopoulos DM, Rigby RA. Generalized additive models for location scale and shape (GAMLSS) in R. J Stat Soft 2007;23:7.

36 Venables WN, Ripley BD. Modern Applied Statistics with S, Fourth Edition. New York: Springer-Verlag, 2002:172-176.

37 Wildiers H, Van CB, van de Poll-Franse LV et al. Relationship between age and axillary lymph node involvement in women with breast cancer. J Clin Oncol 2009;27:2931-2937.

38 Schonberg MA, Marcantonio ER, Li D et al. Breast cancer among the oldest old: tumor characteristics, treatment choices, and survival. J Clin Oncol 2010;28:2038-2045.

39 Vinh-Hung V, Cserni G, Burzykowski T et al. Effect of the number of uninvolved nodes on survival in early breast cancer. Oncol Rep 2003;10:363368 .

40 Vinh-Hung V, Burzykowski T, Cserni G et al. Functional form of the effect of the numbers of axillary nodes on survival in early breast cancer. Int $\mathrm{J}$ Oncol 2003;22:697-704.

41 Vinh-Hung V, Verschraegen C, Promish DI et al. Ratios of involved nodes in early breast cancer. Breast Cancer Res 2004;6:R680-R688.

42 Vinh-Hung V, Storme G. L'envahissement axillaire dans le cancer du sein: un modèle de marche aléatoire. (Abstract 225). Bull Cancer 2006;93:646.

43 Guern AS, Vinh-Hung V. Statistical distribution of involved axillary lymph nodes in breast cancer [in French]. Bull Cancer 2008;95:449-455.

44 Tai P, Cserni G, Van de Steene J et al. Modeling the Effect of Age in T1-2 Breast Cancer using the SEER Database. BMC Cancer 2005;5:130.

45 Danko ME, Bennett KM, Zhai J et al. Improved staging in node-positive breast cancer patients using lymph node ratio: results in 1,788 patients with long-term follow-up. [With Discussion]. J Am Coll Surg 2010;210:797807.

46 American Joint Committee on Cancer, Edge SB, Byrd DR, et al. AJCC Cancer Staging Manual, Seventh Edition. New York: Springer, 2010.

47 Chambers JM, Cleveland WS, Kleiner B et al. Graphical methods for data analysis. Belmont, CA and Boston, MA: Wadsworth International Group and Duxbury Press, 1983:75-128.

48 de Onis M, Onyango A, Borghi E et al. WHO Child Growth Standards. Length/height-for-age, weight-for-age, weight-for-length, weight-forheight and body mass index-for-age. Methods and development. Geneva, Switzerland: WHOPress, World Health Organization, 2006. Available at http:// www.who.int/childgrowth/standards/technical_report/en/index.html. Accessed July 20, 2010.

49 de Onis M, Onyango A, Borghi E et al. WHO Child Growth Standards.
Growth velocity based on weight, length and head circumference. Methods and development. Geneva, Switzerland: WHO Press, World Health Organization, 2009. Available at http://www.who.int/childgrowth/standards/ velocity/technical_report/en/index.html. Accessed July 20, 2010.

50 Khondoker MR, Glasbey CA, Worton BJ. A comparison of parametric and nonparametric methods for normalising cDNA microarray data. Biom J 2007;49:815-823.

51 Mouksassi MS, Marier JF, Cyran J et al. Clinical trial simulations in pediatric patients using realistic covariates: application to teduglutide, a glucagon-like peptide-2 analog in neonates and infants with short-bowel syndrome. Clin Pharmacol Ther 2009;86:667-671.

52 Gorgulu S, Can MF, Yagci G et al. Extracapsular extension is associated with increased ratio of metastatic to examined lymph nodes in axillary node-positive breast cancer. Clin Breast Cancer 2007;7:796-800.

53 Vinh-Hung V, Storme G. No nodal cutoff in node-positive breast cancer women treated with mastectomy. Breast Cancer Res Treat 2006;98:173178.

54 Vinh-Hung V, Bourgain C, Vlastos G et al. Prognostic value of histopathology and trends in cervical cancer: A SEER population study. BMC Cancer 2007;7:164.

55 Vinh-Hung V, Truong PT, Janni W et al. The e;ffect of adjuvant radiotherapy on mortality differs according to primary tumor location in women with node-positive breast cancer. Strahlenther Onkol 2009;185:161-168.

56 Nguyen NP, Ly BH, Betz M et al. Importance of age as a prognostic factor for tonsillar carcinoma. Ann Surg Oncol 2010 (in press).

57 Raftery AE. Bayesian model selection in social research (with discussion by Andrew Gelman \& Donald B. Rubin, and Robert M. Hauser, and a rejoinder) Sociol Methodol 1995;25:111-195.

58 Hoeting JA, Madigan D, Raftery AE et al. Bayesian model averaging: a tutorial (with comments by M. Clyde, David Draper and E. I George, and a rejoinder by the authors). Statist Sci 1999;14:382-417.

59 Efron B, Gous A. Scales of Evidence for Model Selection: Fisher versus Jeffreys. Institute of Mathematical Statistics Lecture Notes-Monograph Series 2001;38:210-246.

60 Walsh L. A short review of model selection techniques for radiation epidemiology. Radiat Environ Biophys 2007;46:205-213.

61 Joslyn SA, Konety BR. Effect of axillary lymphadenectomy on breast carcinoma survival. Breast Cancer Res Treat 2005;91:11-18.

62 Aapro M, Monfardini S, Jirillo A et al. Management of primary and advanced breast cancer in older unfit patients (medical treatment). Cancer Treat Rev 2009;35:503-508.

63 Muss HB, Woolf S, Berry D et al. Adjuvant chemotherapy in older and younger women with lymph node-positive breast cancer. JAMA 2005;293: 1073-1081.

64 Muss HB. Adjuvant treatment of elderly breast cancer patients. Breast 2007;16(Suppl 2):S159-S165.

65 Dellapasqua S, Colleoni M, Castiglione M et al. New criteria for selecting elderly patients for breast cancer adjuvant treatment studies. The Oncologist 2007;12:952-959.

66 Joslyn SA, Sirintrapun SJ, Konety BR. Impact of lymphadenectomy and nodal burden in renal cell carcinoma: retrospective analysis of the National Surveillance, Epidemiology, and End Results database. Urology 2005;65: $675-680$.

67 Centers for Disease Control and Prevention and the Merck Company Foundation. The State of aging and health in America 2007. Whitehouse Station, NJ: The Merck Company Foundation, 2007. Available at http://www.cdc. gov/aging. Accessed January 7, 2010. 
68 Hurria A. Clinical trials in older adults with cancer: past and future. Oncology (Williston Park) 2007;21:351-358.

69 Aapro MS, Kohne CH, Cohen HJ et al. Never too old? Age should not be a barrier to enrollment in cancer clinical trials. The Oncologist 2005; 10:198204.

70 Surbone A, Kagawa-Singer M, Terret C et al. The illness trajectory of elderly cancer patients across cultures: SIOG position paper. Ann Oncol 2007;18:633-638.

71 Muss HB, Berry DA, Cirrincione CT et al. Adjuvant chemotherapy in older women with early-stage breast cancer. N Engl J Med 2009;360:2055-2065.

72 Giordano SH, Duan Z, Kuo YF et al. Use and outcomes of adjuvant chemotherapy in older women with breast cancer. J Clin Oncol 2006;24:27502756.
73 Elkin EB, Hurria A, Mitra N et al. Adjuvant chemotherapy and survival in older women with hormone receptor-negative breast cancer: assessing outcome in a population-based, observational cohort. J Clin Oncol 2006;24: $2757-2764$.

74 Keam B, Im SA, Kim HJ et al. Clinical significance of axillary nodal ratio in stage II/III breast cancer treated with neoadjuvant chemotherapy. Breast Cancer Res Treat 2009;116:153-160.

75 Extermann M. Interaction between comorbidity and cancer. Cancer Control 2007;14:13-22.

76 Gloeckler Ries LA, Reichman ME, Lewis DR et al. Cancer survival and incidence from the Surveillance, Epidemiology, and End Results (SEER) program. The Oncologist 2003;8:541-552. 